\title{
The effect of different concentrations of heparin sealing liquid on thrombosis after peripherally inserted Central Catheter catheterization in tumor patients (HEALTH): protocol for a prospective, single-blind, randomized, controlled trial
}

\section{Zhimin Wang}

Guangzhou First Municipal Peoples Hospital

Peiyi He ( $\nabla$ pyhe0610@163.com )

Guangzhou First Municipal Peoples Hospital https://orcid.org/0000-0002-5977-8867

Jianning Wei

Guangzhou First Municipal Peoples Hospital

\section{Ping Du}

Guangzhou First Municipal Peoples Hospital

\section{Method Article}

Keywords:

Posted Date: November 30th, 2018

DOI: https://doi.org/10.21203/rs.2.54/v1

License: (9) This work is licensed under a Creative Commons Attribution 4.0 International License. Read Full License 


\section{Abstract}

Background: Malignant tumor patients with peripherally inserted central catheter (PICC) are at high risk of developing deep venous thrombosis. Different concentrations of sealing liquid result in different outcomes. However, there is a lack of evidence of large randomized controlled trials to show the different incidences of deep vein thrombosis using different concentrations of sealing liquid. The aim of this study is to compare the difference in the incidence of deep vein thrombosis of the upper extremity in cancer patients with PICC using physiological saline, $10 \mathrm{U} / \mathrm{ml}$ heparin, and $50 \mathrm{U} / \mathrm{ml}$ heparin, separately.

Methods: A three-arm, single-center, single-blind, randomized controlled trial will be performed. We plan to recruit a total of 639 cancer patients within 12 months with a follow-up period of six months. Patients will be randomized at 1:1:1 ratio by centralized service allocation concealment. Sealing liquid with different concentrations, i.e. physiological saline, $10 \mathrm{U} / \mathrm{ml}$ heparin, and $50 \mathrm{U} / \mathrm{ml}$ heparin will be administered. Primary outcome is the incidence of upper extremity venous thrombosis. Secondary outcomes include the time of thrombus and the severity of thrombosis with three grades. All randomized patients will be analyzed by intention-to-treat. Chi-square test will be used to compare the incidence of upper extremity venous thrombosis. Kaplan-Meier survival curve and log-rank test will be presented to compare thrombus in relation to time. A Cox regression will test the effect of group on thrombus, with hazard ratio adjusted for significant clinical variables.

Discussion: The trial will help to resolve the uncertainty of the clinical practice of PICC catheterization liquid concentration, and to provide the basis for the implementing the clinical intervention and effectively reducing PICC-related venous thrombosis. The present study is ongoing, and data will be published in peer-reviewed journals after its conclusion.

Trial registration: Chinese Clinical Trial Registry, ChiCTR1800018391. Registered on 14 September 2018 Retrospectively registered, http://www.chictr.org.cn/showproj.aspx?proj=30808.

\section{Background}

Peripherally inserted Central Catheter (PICC) is a method of catheterization of the peripheral vein (the main vein, the median vein of the elbow, the cephalic vein, etc.), so that the tip of the catheter is located in the superior vena cava [1]. PICC was introduced to China in the late 1990s and is now widely used in cancer intravenous chemotherapy, long-term infusion, total parenteral nutrition (TPN), and the construction of nutritional pathways in preterm infants [2-5]. Although the placement of the PICC can produce fewer complications than other central venous catheters, as a foreign body in the venous lumen, PICC also could result in complications, such as phlebitis, infection, catheter blockage and thrombosis. Among them, catheter-related venous thrombosis is a serious complication [7]. PICC-related venous thrombosis refers to the process of forming a blood clot in the inner wall of the vessel and the wall of the catheter where the PICC is located due to multiple factors such as puncture or direct injury of the intima 
of the vessel and the patient's own state after PICC catheterization [8]. The incidence of PICC-related deep venous thrombosis varies greatly depending on the population, screening methods, and catheter type. The incidence of pulmonary embolism caused by upper extremity venous thrombosis is $35 \%$ [10], and the incidence of tumor patients is more than twice that of the general population, reaching $51.4 \%[11,21,23]$. The main risk factors included individual patient factors, catheter placement, and catheter-related factors $[11,21]$. Therefore, how to effectively prevent PICC thrombosis is the focus of clinical nursing work. It is
also an urgent problem to be solved.

Sealing is one of the basic conditions to ensure the smoothness of the PICC catheter. When the infusion is completed or is during the intermittent period, the tube should be sealed once a week to ensure smooth drainage. According to the "Intravenous Therapy Technical Practices" implemented in China since May 1 , 2014 , the maintenance of intravenous catheters requires sealing with the use of a saline or heparin saline, the volume of which should be twice the total volume of a catheter plus an extension tube, and with positive pressure. The concentration of heparin saline can be $100 \mathrm{U} / \mathrm{ml}$ in the infusion port, or from 0 to $10 \mathrm{U} / \mathrm{ml}$ in PICC and CVC. Since January 2014, we have used $0.9 \%$ saline as a sealing liquid for patients with cancer in our center with indwelling three-way membrane PICC. $10 \mathrm{U} / \mathrm{ml}$ heparin saline was used as a sealing liquid for patients with terminal open PICC. The results showed that the incidence of catheterrelated venous thrombosis was higher, at $29.17 \%$ [24], which increased the number of hospital stays and treatment costs. Studies of the meta-analysis of the effectiveness and safety of heparin saline and saline have shown that heparin saline was more effective than saline in reducing the rate of venous catheter occlusion and in preventing the formation of venous thrombosis [25], but the concentration of heparin solution was not studied in the research. The study from Yang and colleagues[26] reported that the use of high-concentration heparin saline (heparin $50 \mathrm{mg}$ plus saline $4 \mathrm{ml}$ ) sealed tube can effectively prevent deep vein thrombosis caused by femoral vein catheterization, with no significant increase in the bleeding rate. Patients with malignant tumors are hypercoagulable and are at high risk of venous thrombosis. However, there is insufficient evidence on this population. Therefore, it is necessary to evaluate the difference of thrombus formation in PICC tumor patients with different concentrations of heparin sealing liquid, to provide a scientific basis for the prevention of thrombosis by PICC sealing with the use of sealing liquid in different concentrations.

The current standard for the use of normal saline and heparin concentrations is based on the 2011 edition of the American Infusion Association (INS) guidelines and the Code of Practice for Intravenous Therapy Care Technology, which was implemented on May 1, 2014 in China. Maintenance of the catheter requires the use of normal saline the volume of which is twice the total volume of a catheter plus an extension tube or a $10 \mathrm{U} / \mathrm{ML}$ heparin saline positive pressure seal. However, there is no uniform standard in practical applications. At present, the most commonly used methods for sealing liquid concentration in China and other countries have their own advantages and disadvantages. These include (1) saline sealing tube: when sealing the tube solution, it is not restricted by disease type, especially for patients with hemorrhagic tendency, coagulation mechanism disorder and liver and kidney dysfunction. Normal saline, which is now widely used in clinical practice, is a $0.9 \%$ sodium chloride solution the osmotic pressure of which is equal to that of the human plasma. However, Jonker et al [27] found that saline as a 
sealing liquid also has its shortcomings, because physiological saline has no anticoagulant effect, although it can play a role in maintaining catheter patency, it also increases the probability of thrombosis. (2) Heparin brine seal: Heparin has strong physicochemical properties with strong negative charge, and can effectively resist blood coagulation in vivo and in vitro, mainly through the interaction with antithrombin III (AT-III) in plasma. AT-III is an inhibitor of activated coagulation factors XIla, Xla, Xa, IXa and thrombin (factor Ila). Heparin can catalyze the interaction of AT-III with many coagulation factors, prevent the aggregation and destruction of the platelet, and impede the formation of Thrombin; prevent prothrombin from becoming thrombin; inhibit thrombin, and prevent fibrinogen from becoming fibrin, thereby exerting anticoagulant effects. Because of its strong anti-coagulation effect in vivo and in vitro, and low toxicity, a small amount of heparin solution has no effect on the human body, so it is used to prevent the serious complications of post-tube thrombosis [28]. The main adverse reaction of heparin is that excessive use of the drug can cause spontaneous bleeding. The long-term use of heparin by patients with Hepatic insufficiency can cause thrombocytosis tendency due to depletion of antithrombin-III. And the use of heparin is prohibited in Heparin-induced thrombocytopenia (HIT).

Studies in China have shown that different types and concentrations of sealing liquids have different effects in tube formation, catheter patency and thrombosis. A controlled trial conducted by Zhang et al [17] showed that PICC-negative tumor patients were sealed with three concentrations $(50,125,250 \mathrm{U} / \mathrm{ml})$ of heparin dilution, and the incidence of blockage was $70 \%, 40 \%$, and $15 \%$, respectively. Two different concentrations and doses of heparin sealing liquid were compared by $\mathrm{Yu}$ and colleagues[19]. It was found that inpatients who received intravenous indwelling needles were best treated with $50 \mathrm{U} / \mathrm{mL}$ heparin solution to maintain venous catheter patency and reduce extubation rate. Xiao et al [20] did a 1:1 parallel randomized trial among patients with PICC-negative nasopharyngeal carcinoma in T3-T4 stage. One group was sealed with $20 \mathrm{ml}$ of normal saline pulse positive pressure, and the other group was sealed with $20 \mathrm{ml}$ of $20 \mathrm{U} / \mathrm{mL}$ heparin dilution. There was a significant difference in thrombus formation rates between the two groups, with the rate of the heparin group lower than that of the saline group $(11.4 \%$ vs $48.6 \%)$. Zeng et al. conducted a systematic review of the effects of two different sealing liquids in prevention of PICC thrombosis and catheter occlusion in China. The results of five randomized controlled trials showed that the concentration range was $10 \mathrm{U} / \mathrm{ml}-125 \mathrm{U} / \mathrm{ml}$ for the heparin group. The effect of preventing PICC catheter thrombosis and tube occlusion is better in the group sealing with heparin than the group with sterile saline [22].

However, the literature review from the Cochrane System Review database [14] showed that there was no significant statistical difference in the incidence of infection and mortality between adult patients undergoing CVC (central venous catheters) catheterization sealed using $0.9 \%$ saline and $0.9 \%$ heparin solution. There was consistency in patency rate between Leukemia patients undergoing PICC catheterization in a home environment using a $100 \mathrm{U} / \mathrm{ml}$ catheter and a $10 \mathrm{U} / \mathrm{ml}$ heparin solution in sealing tube [15]. Another RCT study showed that there was also no statistically significant difference in the patency rate between hemodialysis patients using $0.9 \%$ saline and $1000 \mathrm{IU}$ heparin [16]. 
In summary, there is a difference among the studies' results from the population and treatment therapies. In China, there is also a lack evidence of large randomized controlled trial to show the difference. This project studies the question existing in the above research in China and abroad. In this study, PIMC patients with malignant tumors would be sealed with different concentrations of sealing liquid in three different groups in a 1:1:1 randomized controlled trial to compare the difference in the incidence of deep vein thrombosis of the upper extremity in tumors patients with PICC using physiological saline, $10 \mathrm{U} / \mathrm{ml}$ heparin solution, and $50 \mathrm{U} / \mathrm{ml}$ heparin solution. Thrombosis would be screened at different catheter indwelling time, with follow-up observation until extubation, to investigate the exact occurrence and differences of PICC-related upper extremity venous thrombosis in different groups of sealing liquids, and to provide guidance for clinical intervention and effective reduction of thrombosis rate.

\section{Methods/design}

\section{Trial design}

This study is prospective, single-center, single-blind, randomized, 1:1:1 parallel controlled trial, aiming at comparing the difference in the incidence of upper extremity venous thrombosis in tumor patients with PICC using physiological saline, $10 \mathrm{U} / \mathrm{ml}$ heparin solution, and $50 \mathrm{U} / \mathrm{ml}$ heparin solution. The study will respect the Consolidated Standards of Reporting Trials (CONSORT) as show in the Figure 1 with the content for the schedule of enrolment, interventions and assessments. The study will also respect the Standard Protocol Items: Recommendations for Interventional Trials (SPIRIT) as shown in Additional file 1 , respectively.

\section{Hypothesis}

This study has three research hypotheses:

First, there is a statistical significance in comparing the difference of the incidence of thrombus between the saline group and the $10 \mathrm{U} / \mathrm{ml}$ heparin solution group (first main hypothesis).

Second, there is a statistical significance in comparing the difference of the incidence of thrombus between the saline group and the $50 \mathrm{U} / \mathrm{ml}$ heparin solution group (second main hypothesis).

Third, there is a statistical significance in comparing the difference of the incidence of thrombus between the $10 \mathrm{U} / \mathrm{ml}$ heparin solution group and the $50 \mathrm{U} / \mathrm{ml}$ heparin solution group (third main hypothesis).

Setting and sample (population)

Selection of research subjects

About 639 patients with PICC will receive one of the three different concentrations of the sealing liquid in the Department of Oncology, Guangzhou First People's Hospital. The subjects will be randomly assigned in a ratio of 1:1:1 to receive one of the three concentrations of the sealing liquid. The enrollment period 
was from July 2017 to June 2018, and patients with malignant tumors who were placed in PICC at the First People's Hospital of Guangzhou will be screened. Patients who meet the following inclusion criteria and no exclusion criteria listed in Table 1 will be eligible. Patients who have serious adverse events or unplanned extubation will be exit. All subjects who have signed an informed consent form and screened for entry into the trial have the right to withdraw from the trial at any time. Those who do not complete the observation cycle specified by the protocol will be reported.

\section{Informed Consent:}

Informed consent forms will be signed by tumor patients who are placed in PICC in the First People's Hospital of Guangzhou and who meet the criteria listed in Table1.

Outcome measures and definitions

\section{Primary outcome}

The primary outcome is incidence of upper extremity venous thrombosis (\%). The term is defined as the rate of patients with upper extremity venous thrombosis who were placed in a PICC. The Denominator of the rate is number of cases that have been placed in a PICC per unit time. The Numerator is the number of cases of thrombosis happened in lateral upper limb per unit time.

\section{Secondary outcomes}

\section{The time of thrombus}

The time of thrombus is defined as the time after replacement the time of diagnosis of thrombosis by color Doppler ultrasound.

2. The severity of thrombosis, including grade I, grade II, and grade III. It is defined as:

1) Grade I: After the catheter is placed in the venous lumen, there is a small hill-shaped mass echo or a small agglomeration echo outside the catheter, mainly in isolated type. It is examined by color Doppler flow imaging, and the venous blood flow is smoothly presented. There is a 1-30\% narrow surface. Under direct vision, it can be found that there are lower echoes in the blood vessels and the wall, and the blood flow is small, and the color blood flow cannot reach full filling.

2) Grade II: Through examination, it is found that there is a thrombus formation around the catheter or in the venous lumen, and there are many places. It is found by CDFI that there is relatively unobstructed venous blood flow, and there is a narrow surface of $31-50 \%$ in the cross section of the blood vessel. It can be seen that the amount of echo in the blood vessel and the lower wall is moderate, and the color vessel filling state has a relatively serious defect.

3) Grade III: The thrombus is completely blocked, and the thrombus around the catheter and the venous cavity is more common, mainly of the fusion type. The venous thrombosis is mainly composed of fusion, 
and is presented in a large piece. The thrombus is basically filled with a large area tube. The cavity, through CDFI examination, found that there is no blood flow signal or a small amount of blood flow signal in the narrow channel, and the cross section of the blood vessel has more than $50 \%$ of the narrow surface. Under direct vision, there are more mid-echo and low echo in the blood vessel. The blood cannot present a colored blood flow in the blood vessels and cannot collapse the blood vessel wall.

\section{Adverse Events:}

The incidence of adverse reactions will be assessed among three groups. Adverse reactions include bleeding and coagulopathy. Adverse reactions were based on activated partial thromboplatin time (aPTT), prothrombin time (PT), fibrinogen (Fbg), and plasma thrombin time (TT) to assess whether the patient's coagulation function is normal, with or without bleeding tendency. Serious adverse reactions will be monitored and reported to the Ethics Committee in a timely manner.

Recruitment, randomization, allocation concealment and blinding

Since the catheterization was mainly performed during working hours, this study used a full-time research nurse to screen patients. For each patient who receives informed consent, the research nurse will train them and their families to fully understand the research protocol. After the patient has obtained informed consent, the research nurse will contact the randomizer of the trial to obtain a random number to which study group for the patient. The randomization scheme is generated by the SAS statistical analysis software random grouping module, and the random number is stored in an envelope and managed by the research randomizer. Patients will be randomized to a 1:1:1 ratio, 1) saline group, 2) $10 \mathrm{U} / \mathrm{ml}$ heparin solution group, 3) $50 \mathrm{U} / \mathrm{ml}$ heparin solution group. This study was set up as single blind, i.e. the patient did not know the concentration of the sealing liquid used. Because in the research nurses need to know the concentration of the sealing liquid used during the operation, it is difficult that the nurses are blinded. But statistician will be blinded.

\section{Procedure}

The study includes four stages. They are 1) Pre-screening stage: to make PICC catheterization for tumor

patients; 2) Screening period: to confirm whether the subject meets the study qualification. This stage begins after the signing of the informed consent form (ICF) and continues until the random assignment (up to 2 weeks); 3 ) Intervention period: from the time of randomization to the occurrence of upper extremity venous thrombosis. Participant will be given different concentrations of sealing liquid to seal the tube; 4) Follow-up period: Start from the first visit (defined as the first day when the subject receives the dispensed sealant); perform a vascular ultrasound every week for the first month, and thereafter every month A vascular ultrasound is performed until a thrombus occurs or the study is over. In addition, the last vascular ultrasound was performed on the day before extubation of the subject to assess whether the subject developed a thrombus. 
Interventions Sealing: In addition to the three concentrations of the three sealing liquids, all the other operations follow the same standardized operating procedures.

1) Time of sealing

After each infusion, the tube is first flushed and then sealed; in the intermittent period, the tube is flushed and sealed once a week.

2) Sealing methods

A. saline sealing: SAS (S, saline; A, administration; $S$, saline)

B. $10 \mathrm{U} / \mathrm{ml}$ heparin saline sealing: SASH (S physiological saline; A administration; S physiological saline; $\mathrm{H}$ heparin saline)

C. $50 \mathrm{U} / \mathrm{ml}$ heparin saline: $\mathrm{SASH}$ (S saline; A administration; $\mathrm{S}$ saline; $\mathrm{H}$ heparin saline)

3) Sealing liquid volume

Sealing liquid volume $=$ (catheter volume + additional device volume $) \times 2$

4) Sealing methods: pulsed flush the tube and then seal it with positive pressure

(1) flush tube with pulse: use $0.9 \%$ saline $10-20 \mathrm{ml}$ and push-stop-push bolus method, so that the physiological saline could form a vortex in the catheter, and the residual drug in the catheter could be rinsed clean;

(2) seal tube with positive pressure: flush tube with $0.9 \%$ saline or heparin saline $5 \mathrm{ml}$ with positive pressure, when the syringe remains $0.5-1 \mathrm{ml}$ sealing liquids, push while pushing the needle until it is completely withdrawn

\section{PICC puncture placement and maintenance methods:}

1) Each patient signed an informed consent form before the PICC was placed.

2) Measure the base arm circumference at $10 \mathrm{~cm}$ above the elbow joint of the upper limb where the tube was placed, and use the color Doppler ultrasonography to examine the condition of the blood vessel to be punctured, including the diameter, elasticity, valve, blood flow velocity, blood vessel curvature, and vessel wall thickness of the blood vessel.

3) Traditional blind puncture method: the patient takes a supine position, and the upper limb and the trunk are stretched by $90^{\circ}$. Measure the total length from the puncture point to the right sterno-lock joint and then down to the third intercostal space, which is the catheter placement length; disinfect the upper arm skin to establish a maximized sterile area; The assistant assists in preparing tools to be used in the placement of the tube and placing them on the sterile table, pre-flushing the catheter, and preparing tools 
for the puncturing. The venipuncture was performed at an angle of $15^{\circ}-30^{\circ}$. After returning blood, the blood vessel was inserted into the puncture sheath in parallel with the blood vessel and the needle core was withdrawn; The catheter with a predetermined length was inserted along the puncture sheath, and the guide wire was removed; the chest piece was taken to determine the position of the catheter.

4) B-ultrasound guided by Seldinger technology: Place a sterile towel to create a maximized sterile area. Apply the aseptic mixture to the B-ultrasound probe with the help of the assistant, and put on the sterile probe cover. Perform the cross-sectional scan under B-ultrasound, and find the thickest vein in the lower third of the venous side. Note that the vein should be distinguished from the artery, that the vein is easily crushed. Move the probe, place the vein of the cross section in the center of the horizontal scale, select different guide needles according to the depth, put the needle into the guide needle, and repeat the previous positioning. Hold the probe with your left hand, insert the needle with your right hand, and look at the B-ultrasound interface. When you see the needle tip enters the blood vessel, you will see blood flow from the top of the puncture needle. Remove the B-ultrasound, place the guide wire along the puncture needle, and remove the puncture needle when it is placed approximately $20-30 \mathrm{~cm}$. At the puncture point, perform local anesthesia by injecting lidocaine, use the knife to expand the skin. The cutting depth of the cutting knife was determined according to the fatness of the patient. Insert the guiding sheath along the guide wire, then withdraw the guide wire and introduce the sheath core. Insert the PICC into the vein along the guiding sheath to the same length of the preset tube, then remove the guide wire; determine the position of the catheter using the chest piece.

5) Immediately after catheterization, check whether the blood vessels placed in the PICC catheter have thrombosis by performing color Doppler ultrasound, and record relevant information.

6) PICC maintenance: including replacement of the applicator and heparin cap. $10 \mathrm{ml}$ Pulse tube with positive pressure sealing tube by using a $0.9 \%$ injection of physiological saline, $1-2$ times a week.

\section{Selection of catheters and Seldinger puncture bags:}

Catheters: the 4Fr single-chamber three-way valve PICC and the open-end high-pressure single-chamber PICC from Bard. Seldinger puncture bags: the MST puncture kit from Bard.

\section{Research equipment:}

Ultrasound used in Seldinger technology: U.S. Sonor's L25 two-dimensional real-time portable bedside Doppler color ultrasound diagnostic instrument and Site-Rite $5 \mathrm{~B}$ ultrasound guidance system produced by Bard. The $5-10 \mathrm{MHz}$ broadband probe was used.

Confirmed thrombosis B-ultrasound: IE33 color ultrasonic diagnostic instrument produced by Philips, USA. The probe is $10 \mathrm{~L}$.

Data collection and storage 


\section{Baseline Information}

Measurement indicators: This report table mainly collects the patient's social demographic data: including the patient's name, age, gender, education level, occupation, payment method of medical expenses, lifestyle, etc; Medical history data: including diagnosis, pathological findings, comorbidities, history of surgery, history of thrombosis, history of trauma, family history, history of smoking, history of central venous catheterization, history of PICC catheterization, usual activity, number and protocols of radiotherapy and chemotherapy, etc; Patient catheterization: including catheter placement, puncture site, number of punctures, veins placed, catheter tip position, etc. Laboratory testing, including white blood cell (WBC), red blood cell (RBC), hemoglobin (HGB), platelet count (PLT), Prothrombin time (PT), activated partial thromboplastin time (aPTT), fibrin(-ogen) degradation product (FDP), fibrinogen (Fbg), D-dimer, blood glucose, total cholesterol, triglycerides, liver function and other indicators, as well as monitored veins, symptoms and signs, B-ultrasound results for each examination, diagnostic criteria for thrombosis, etc. The number of days the catheter and Eastern Cooperative Oncology Group (ECOG) score will be recorded as well.

\section{Follow-up}

1) Follow-up time: From the time the patient is placed into the PICC until the catheter is removed. The longest follow-up period was 1.5 years and the shortest follow-up was half a year.

2) Follow-up information:

(1) Vascular ultrasonography will be performed at 1, 2, 3, and 4 weeks after catheterization, and then a vascular ultrasonography will be performed every 4 weeks to screen for thrombosis, and the time and grade of thrombosis will be recorded until the catheter is removed.

(2) Patients at least once a week to return to the hospital for catheter maintenance and assessment of venous thrombosis symptoms, including the patient consciously perceives the side limbs, armpits, shoulder and arm pain, and the upper limbs appear red, swollen, hot, pain and other symptoms, and the increase of the arm circumference compared with when the tube was placed. At the same time, any telephone consultation from the patient and returning to the hospital to ask about the condition would be dealt with immediately.

Case Report Forms will be stored in the trial unit of the research center during the trial, after trial. The research data will be entered into a database created by Epidata software and managed by a dedicated data administrator.

Sample size and power calculation

According to the study hypotheses, we planned to recruit a total of 639 PICC patients with malignancy, and randomize into three groups with 1:1:1 ratio. The main purpose of this study was to prove the difference of incidence of upper extremity venous thrombosis among these three groups. On the basis of 
previous report [11] and the preliminary study in our center [24], we assumed that the incidence of upper extremity venous thrombosis was $30-40 \%$ in the saline group, $25 \%$ in the $10 \mathrm{U} / \mathrm{ml}$ heparin solution group, and $15 \%$ in the $50 \mathrm{U} / \mathrm{ml}$ heparin solution group. To test the first main hypothesis of the difference of the incidence of thrombus between the saline group and the $10 \mathrm{U} / \mathrm{ml}$ heparin solution group, we estimated that we would need a total of 639 participants with 213 at each group to achieve $80 \%$ power at a twosided $1.7 \%$ (5\%/3, Bonferroni method) significance level, with an enrolment period of one year and a follow up period of a minimum of half a year, and taking into account the $5 \%$ dropout rate. Under these sample size, we have as least $99.99 \%$ and $72 \%$ power to test the other two hypotheses (second main hypothesis, third main hypothesis, respectively). We did not plan to do an interim analysis.

Statistical rationale and analysis plan

\section{Study population}

All randomised patients will be analysed by intention-to-treat (ITT). Assessment for baseline characteristics will be analysed using full analysis set. Efficacy assessment will be analysed by full analysis set and per protocol set. Safety analysis will use safety set including patients receiving PICC in the study.

\section{Statistical analysis}

All data analyses will be conducted using SAS 9.4 statistical software. Missing data will not be imputed. An as-treated analysis will assess the effect of protocol violations. Baseline descriptive characteristics will be presented using means and SD or medians and interquartile range (IQR) for continuous variables and counts/percentages for categorical variables. ANOVA or Kruskal-Wallis test will used to compare continuous variables of baseline characteristics, radiological features and experimental data and multiple comparisons between two groups will be performed using $t$ test or Wilcoxon test. Ordinal categorical data will be compared using Kruskal-Wallis test among three groups and Wilcoxon test between two groups. Non-ordinal categorical variables will be compared using Chi-square test or Fisher exact test. Primary analysis will be the comparison of incidence of upper extremity venous thrombosis using Chi-square test. Kaplan-Meier survival curves will be presented to compare group failure in relation to dwell time, and the log-rank test will be used to compare the survival curves between study groups. A Cox regression will test the effect of group on failure, with hazard ratios calculated and adjustment for significant patient, device and clinical variables. No subgroup analysis and interim analysis will be performed in the data analysis. Due to the short period of trial, we did not set data monitoring committee during the trial procedure. $\mathrm{P}$ values $<0.05$ will be statistically significant. The study statistician will be blinded.

\section{Discussion}

PICC catheterization is widely used in clinical practice. Catheter-related venous thrombosis is a serious complication. Effective prevention of PICC thrombosis is the focus of clinical nursing work. Sealing is one of the basic conditions to ensure the smoothness of PICC catheters. It has been reported that different 
concentrations of heparin sealing liquids may have different effects on thrombosis in PICC patients with cancer. However, at present, in China it is still controversial about what kind of concentration sealing liquid should be used for PICC catheterized patients.

This RCT study evaluates the difference in thrombus formation between PICC patients with cancer using different concentrations of heparin sealing liquids, which will help to resolve the uncertainty of the clinical practice of PICC catheterization liquid concentration, and to provide the basis for the implementing the clinical intervention and effectively reducing PICC-related venous thrombosis.

The study has some limitations. According to the hypothesis that there is a statistically significant difference in the incidence of thrombosis between the $50 \mathrm{U} / \mathrm{ml}$ heparin solution group and the $10 \mathrm{U} / \mathrm{ml}$ heparin solution group, this study shows that only $72 \%$ of the tests could detect the difference. In this study, the sample size is large when the first and second main research hypotheses are met. However, to make the hypothesis achieve more than $80 \%$ of the third main hypothesis, a larger sample is needed, and it is hoped that more samples can be collected for verification. In addition, the study was set to be singleblind because the nurses were unable to do blindness due to actual operational needs. However, in order to avoid bias, we conducted uniform training for the daily maintenance of the catheter, including the operation procedures such as flushing, sealing, changing dressings and infusion joints. After the training, research nurses must pass two tests to do the work. According to the CDC Guidelines for Prevention of Intravascular Catheter-Related Bloodstream Infections, the relevant procedures and guidelines for PICC care have been developed and improved. Finally, this is a single-center study and hopes to be multicentered in the future.

\section{Declarations}

\section{Trial status}

Patient recruitment is currently underway according to the protocol version 1.0. The study started at 4th Jan 2018 and is expected to end at 1st July 2018.

\section{List of abbreviations}

PICC: peripherally inserted central catheter; TPN: total parenteral nutrition; RCT: randomized controlled trial; INS: American Infusion Association; AT-III: antithrombin III; HIT: Heparin-induced thrombocytopenia; CVC: central venous catheters; CONSORT: Consolidated Standards of Reporting Trials; SPIRIT: Standard Protocol Items: Recommendations for Interventional Trials; ICF: informed consent form; SAS: saline, administration, saline; SASH: physiological saline, administration, physiological saline, heparin saline; WBC: white blood cell; RBC: red blood cell, HGB: hemoglobin; PLT: platelet count; PT: Prothrombin time; aPTT: activated partial thromboplastin time; FDP: fibrin(-ogen) degradation product; Fbg: fibrinogen; ECOG: Eastern Cooperative Oncology Group; ITT: intention-to-treat; IQR: interquartile range; TT: plasma thrombin time. 


\section{Ethics approval and consent to participate}

All study protocols were reviewed and approved by the Ethics Committee of Guangzhou People's Hospital (ethical approval number K2016-089-01). Informed consent will be obtained from all study participants.

\section{Consent for publication}

Not applicable

\section{Availability of data and material}

The datasets generated and/or analysed during the current study are not publicly available due to incompleteness of the study but will be available from the web-based medical research public management platform (ResMan; weblink: http://www.medresman.org/login.aspx) in China after six months of the date the study has completed.

\section{Competing interests}

The authors declare that they have no competing interests

\section{Funding}

This work is supported by grants from the Guangdong Provincial Medical Science and Technology Research Fund (2016111181942734 A2017061). The funder had no role in study design, data collection and analysis, decision to publish or preparation of the manuscript.

\section{Authors' contributions}

ZMW, PYH conceived the study. ZMW, HPY designed the protocol. ZMW, PYH, JNW, and PD secured funding. ZMW wrote the first draft of the manuscript. PYH wrote the final version of the manuscript. All authors contributed to and approved the final version of the manuscript.

\section{Acknowledgements}

The investigators thank all patients participated in the trial design and recruitment.

\section{Authors' information}

1.Department of Oncology, Guangzhou First Municipal Peoples Hospital, Guangzhou, China, 510180; 2.Department of Thoracic surgery, Guangzhou First Municipal Peoples Hospital, Guangzhou, China, 510180.

\section{References}


[1] Zhong HZ, Zhang ZL. Intravenous infusion therapy nursing. Version 2. Beijing: People's Military Medical Press. 2011; 1:321.

[2] Wang JR. Infusion therapy nursing care practice guidelines and implementation rules. Beijing: People's Military Medical Press. 2010; 21.

[3] Wang YH. Application and nursing of peripherally inserted central venous catheter in tumor chemotherapy. General nursing. 2010; 8(6B): 1547-1548.

[4] Lai XL, Wang YQ, Li PY. Clinical observation and nursing of peripheral venous catheter for tumor chemotherapy. General Nursing.2010; 8(2A): 289-290.

[5] Infusion Nurses Society Infusion Nursing standards of practice. J Infus. 2006; 29(1 Supple):S1-S92】

[6] Wilson TJ, Brown DL, Meurer WJ, et al. Risk factors associated with peripherally inserted central venous catheter-related large vein thrombosis in neurological intensive care patients Intensive Care Med. 2012; 38(2):272-278.

[7] Sperry BW, Roskos M, Oskoui R. The effect of laterality on venous thromboembolism formation after peripherally inserted central catheter placement. J Vasc Access. 2012; 13(1):91-95.

[8] Luciani A, Clement O, Halimin P, et al. Catheter-relate upper extremity deep venous thrombosis in cancer patients: a prospective study based on Doppler US. Radiology. 2001; 220(3):655-660.

[9] Lin L, Yang BG, Tang HX. Evaluation of PICC upper extremity venous thrombosis by color Doppler ultrasound. Chinese Journal of General Surgery. 2012; 6: 671-674.

[10] Ong B, Gibbs H, Catchpole I, et al. Peripherally inserted central cathetersand upper extremity deep vein thrombosis. Australasian Radiology. 2006; 50(5):451-454.

[11] Liu Y, Gao Y, Wei L, et al. Peripherally inserted central catheter thrombosis incidence and risk factors in cancer patients: A double-center prospective investigation. Therapeutics and Clinical Risk Management. 2015; 11:153-160.

[12] Wang R, Luo O, He L, et al. Preservative-free $0.9 \%$ sodium chloride for flushing and locking peripheral intravenous access device: a prospective controlled trial. J Evid Based Med. 2012;5:205-208.

[13] Li J, Fan YY, Xin MZ, et al. A randomised, controlled trial comparing the long-term effects of peripherally inserted central catheter placement in chemotherapy patients using B-mode ultrasound with modified Seldinger technique versus blind puncture. Eur J OncolNurs. 2014; 18(1), 94-103.

[14] Lopez-Briz E, Ruiz Garcia V, Cabello JB, et al. Heparin versus $0.9 \%$ sodium chloride intermittent flushing for prevention of occlusion in central venous catheters in adults. Cochrane Database SystRev. 2014; (10):Cd008462. 
[15] Andersen KM, Holland, JS. Maintaining the patency of peripherally inserted central catheters with 10 units/cc heparin. J Intraven Nurs. 1992; 15(2):84-88.

[16] Beigi AA, HadiZadeh MS, Salimi F, et al. Heparin compared with normal saline to maintain patency of permanent double lumen hemodialysis catheters: A randomized controlled trial. Adv Biomed Re. 2014; 3:121.

[17] Zhang YX, Wang XY, Wang RM, et al. Observation on the concentration effect of three PICC heparin sealing liquids. Qilu Nursing Journal. 2011; v17 No.229(01):75-76.

[18] Yin WW, Zhang J, Jiang YM, et al. Clinical application of sealing liquid in venous catheterization. Chongqing Medical Journal. 2014; v43(33): 4544-4546.

[19] Yu DL Wang Y, Teng WZ, et al. Observation on the effect of two different concentrations and amounts of heparin sealing liquid on the patency of intravenous indwelling needle. Nursing Practice and Research. 2008; 5(8): 20-21.

[20] Xiao CQ, Li QM, Fan YY, et al. Comparative study of PICC catheterization of two kinds of sealing liquids to prevent venous thrombosis. Home Nurse. 2007; No.66(09):1-2.

[21] Crawford JD, Liem TK, Moneta GL. Management of catheter-associated upper extremity deep venous thrombosis. Journal of Vascular Surgery: Venous and Lymphatic Disorders. 2016; 4(3):375-9.

[22] Su L, Gao Ly. Treatment strategies and preventive measures of indwelling PICC with venous thromboembolism (VTE) in patients with lung cancer chemotherapy.Xinjiang Medical Journal. 2014; 9:97100.

[23] Fallouh N, McGuirk, HM, Flanders SA, et al. Peripherally inserted central catheter-associated deep vein thrombosis: A narrative review. American Journal of Medicine. 2015; 128(7):722-738.

[24] Wei JN, He PY, Du P, et al. Prospective study of modified Singer's placement of PICC-related thrombus under the guidance of B-ultrasound. Lingnan Journal of Emergency Medicine. 2015; 2(20): 160- 162.

[25] Gai QY. A Meta-analysis of the effectiveness and safety of two kinds of intravenous indwelling needle sealing liquids .Journal of Nursing. 2012; 19(7):39-43.

[26] Yang SY. Therapeutic effect of high concentration heparin saline sealing combined with intraductal infusion on prevention of deep venous thrombosis of lower extremity during hemoperfusion. Medical Information. 2016; 29(11):104-105.

[27] Jonker MA, Osterby KR, Vermeulen LC, et al. Does low dose heparin maintain central venous access device patency: a comparison of heparin versus saline during a period of heparin shortage. JPEN. 2010; 34(4): 444-449. 
[28] Li JT, Jiang WD, Sang GW, et al. Clinical Pharmacology. 3rd ed. Beijing: People's Medical Publishing House. 2007; 1560-1563.

\section{Tables}

\section{Table 1 Inclusion and exclusion criteria}

\begin{tabular}{|c|c|}
\hline Inclusion criteria & Exclusion criteria \\
\hline $\begin{array}{l}\varnothing \text { Patients were over the age of } 18 \text { and volunteered to participate in } \\
\text { the study; } \\
\varnothing \text { Patients were with malignant tumor in pathologic diagnosis, and } \\
\text { blanned to have intravenous chemotherapv: }\end{array}$ & $\begin{array}{l}\varnothing \text { Patients with severe cognitive } \\
\text { impairment who are unable to } \\
\text { cooperate; }\end{array}$ \\
\hline $\begin{array}{l}\varnothing \text { The placing of PICC was practiced by nurses specialized in } \\
\text { intravenous treatment with qualification certificate in the First } \\
\text { People's Hospital of Guangzhou. }\end{array}$ & $\begin{array}{l}\varnothing \text { Patients who did not sign an } \\
\text { informed consent form; }\end{array}$ \\
\hline \multirow[t]{4}{*}{$\begin{array}{l}\varnothing \text { Patients received treatment in our hospital, and the PICC } \\
\text { maintenance was performed in the catheterization clinic of our } \\
\text { hospital during the treatment interval. }\end{array}$} & $\begin{array}{l}\varnothing \text { Patients with serious } \\
\text { complications and other serious } \\
\text { chronic diseases; }\end{array}$ \\
\hline & $\begin{array}{l}\varnothing \text { Patients who were not maintained } \\
\text { in our hospital and were not } \\
\text { able to be tracked; }\end{array}$ \\
\hline & $\begin{array}{l}\varnothing \text { Patients who had thrombosis } \\
\text { immediately after } \\
\text { catheterization; }\end{array}$ \\
\hline & $\begin{array}{l}\varnothing \text { Patients with hypercoagulable } \\
\text { status and patients with open- } \\
\text { ended PICC catheters. }\end{array}$ \\
\hline
\end{tabular}

PICC, peripherally inserted central catheter.

\section{Figures}




\begin{tabular}{|c|c|c|c|c|c|}
\hline \multirow[b]{3}{*}{ Time point: } & \multicolumn{5}{|c|}{ Study period } \\
\hline & \multirow{2}{*}{$\begin{array}{c}\text { Enrollment } \\
\text { T0 } \\
\end{array}$} & \multirow{2}{*}{$\begin{array}{c}\text { Preallocation } \\
\mathrm{T} 1 \\
\end{array}$} & \multirow{2}{*}{$\begin{array}{c}\text { Allocation } \\
\mathrm{T} 2 \\
\end{array}$} & \multicolumn{2}{|c|}{ Postallocation } \\
\hline & & & & intervention & T3 \\
\hline \multicolumn{6}{|l|}{ Enrollment: } \\
\hline \begin{tabular}{|l} 
Eligibility screen \\
\end{tabular} & $\mathrm{x}$ & & & & \\
\hline Informed consent & $\mathrm{x}$ & & & & \\
\hline \begin{tabular}{|l|} 
Allocation \\
\end{tabular} & & $\mathrm{x}$ & $\mathrm{x}$ & & \\
\hline \multicolumn{6}{|l|}{ Interventions: } \\
\hline \begin{tabular}{|l|} 
physiological saline group \\
\end{tabular} & & & & $\mathrm{x}$ & \\
\hline $10 \mathrm{U} / \mathrm{ml}$ heparin solution group & & & & $\mathrm{X}$ & \\
\hline $50 \mathrm{U} / \mathrm{ml}$ heparin solution group & & & & $\mathrm{x}$ & \\
\hline \multicolumn{6}{|l|}{ Assessment*: } \\
\hline \begin{tabular}{|l|} 
Demographic data \\
\end{tabular} & & $\mathrm{x}$ & & & \\
\hline \begin{tabular}{|l|} 
Medical history data \\
\end{tabular} & & $\mathbf{x}$ & & & \\
\hline \begin{tabular}{|l|} 
Laboratory testing \\
\end{tabular} & & $\mathrm{x}$ & & & \\
\hline \begin{tabular}{|l|} 
Image testing \\
\end{tabular} & & $\mathrm{x}$ & & & \\
\hline \begin{tabular}{|l|} 
Patient catheterization \\
\end{tabular} & & & & & $\mathrm{X}$ \\
\hline \begin{tabular}{|l|} 
Vascular ultrasonography \\
\end{tabular} & & & & & $\mathrm{x}$ \\
\hline
\end{tabular}

\section{Figure 1}

Template of content for the schedule of enrolment, interventions, and assessments. T0: baseline variables;T1: preoperative evaluations; T2: allocation; T3: postintervention evaluation. *Detailed information could be found in the part of data collection and storage of the present protocol.

\section{Supplementary Files}

This is a list of supplementary files associated with this preprint. Click to download.

- supplement1.doc 